\title{
Ultrastructure of Mitosis and Spindle Pole Bodies in the Zygomycetous Fungus Coemansia reversa Using Conventional Fixation and Freeze Substitution
}

\author{
R.A. Healy ${ }^{1}$, G.J. Celio ${ }^{2}$, T.K.A. Kumar ${ }^{3}$, R.W. Roberson ${ }^{4}$, and D.J. McLaughlin ${ }^{1}$ \\ 1. Department of Plant Biology, University of Minnesota, Saint Paul, USA. \\ 2. University Imaging Centers, University of Minnesota, Saint Paul, USA. \\ 3. Department of Botany, The Zamorin's Guruvayurappan College, Kerala, India. \\ 4. School of Life Sciences, Arizona State University, Tempe, USA.
}

The purpose of the Assembling the Fungal Tree of Life project (AFTOL) is to better understand the evolutionary history of the Kingdom Fungi using multiple data types, including molecular sequences and ultrastructural characters [1]. Microscopic features such as nuclear division and associated organelles, e.g., spindle pole bodies (SPB), are informative for revealing relationships between many taxonomic groups.

One of the hypotheses tested in the AFTOL project is that multiple losses of the flagellum, a structure found in the basal fungal lineages of Chytridiomycota and Blastocladiomycota, led to the transition to spindle pole bodies lacking centrioles in derived fungi. Zygomycetous fungi, distributed among other basal lineages [2], are critical in testing this hypothesis. However, nuclear division and SPB cycles are incompletely known in these fungi, in part due to the challenges of determining fixation protocols that can penetrate their cell walls and preserve membranes and organelles with enough clarity for observation. Three types of SPB have been reported in zygomycetous fungi from among only two groups: two in Entomophthoromycota [3,4] and one in Mucoromycotina [5]. We investigated mitosis and the SPB cycle in the zygomycetous fungus Coemansia reversa (Kickxellomycotina) using transmission electron microscopy (TEM).

Spores were harvested from flooded 3-week-old cultures and germinated on dialysis membranes on malt extract-yeast extract agar. Hyphae from germinated spores were prepared for TEM by chemical fixation (CF) or cryofixation with freeze substitution (FS). For CF, 4-day-old germinated spores were scraped from membranes and fixed in buffered $2 \%$ glutaraldehyde and $2 \%$ acrolein, then post-fixed in buffered $1 \%$ osmium tetroxide. For FS, membranes with 2- to 3-day-old germinated spores were plunged into liquid propane and placed in substitution solution of $1 \%$ glutaraldehyde and $1 \%$ tannic acid in acetone, followed by post-fixation in $1 \%$ osmium tetroxide. Samples from both methods were embedded in resin and serial sectioned for observation with TEM. These CF and FS methods were also tried on the aerial sporulating phase of $C$. reversa but were unsuccessful.

At interphase the SPB consisted of two components: a cytoplasmic, electron-dense sphere containing a cylindrical structure with microtubules oriented perpendicular to the nucleus, and an intranuclear component appressed to the nuclear envelope. The data indicate that cross sections of the SPB contain a ring of nine microtubules (Figure 1A).

The SPB duplicated early in mitosis and separated on the intact nuclear envelope. Short spindle microtubules formed within the nucleus beneath the extranuclear SPB. The nucleolus formed an extensive band along the nuclear envelope distant from the SPB early in mitosis. Nuclear division appears to be intranuclear with spindle and kinetochore microtubules interspersed with the condensed chromatin. A metaphase plate appears to be absent. The nucleolus retained a peripheral position within 
the nucleus or was in a pocket on the nucleus later in mitosis (Figure 1B). Late stages in nuclear division were not observed.

The results in C. reversa indicate that it possesses a fourth type of zygomycetous SPB, and the third type that suggests a reduced centriolar component in zygomycetous fungi. The ring of nine microtubules in C. reversa closely parallels the centriolar structure of Chytridiomycota and Blastocladiomycota, but the nine microtubules are not paired and the central microtubule is absent in C. reversa. These results strongly support the hypothesis of centriolar loss in the derivation of the SPBs of derived fungi with various degrees of modification of the centriole in different taxa [6].

\section{References:}

[1] DJ McLaughlin et al, Trends in Microbiology 17 (2009), p. 488.

[2] DS Hibbett et al, Mycological Research 111 (2007), p. 509.

[3] LJ McKerracher and IB Heath, Mycologia 77 (1985), p. 412.

[4] TM Butt and A Beckett, Protoplasma 120 (1984), p. 61.

[5] EK McCully and CF Robinow, Archiv für Mikrobiologie 94 (1973), p. 133.

[6] The authors acknowledge funding from the National Science Foundation, NSF award DEB-0732550. We thank Corey Nelson for assistance with cultures. All microscopy was done at the University Imaging Centers at the University of Minnesota.
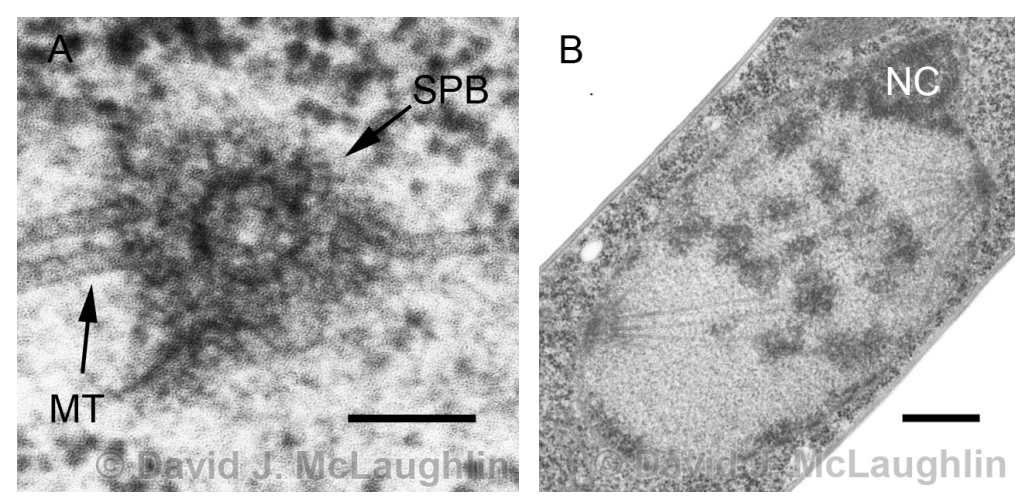

Figure 1. Ultramicrographs of freeze substituted germinated spores of Coemansia reversa. A) Cross section of spindle pole body (SPB) with microtubules (MT). Scale bar $=0.1 \mu \mathrm{m}$. B) Longitudinal section of $C$. reversa hypha with nucleus in metaphase-anaphase. The nucleolus (NC) is in a peripheral pocket of the nucleus. Scale bar $=0.5 \mu \mathrm{m}$. 